\title{
DE NUEVO, LOS HECHOS EN SERIO*
}

\author{
William Twining \\ University College London
}

RESUMEN. Este ensayo es la continuación de un artículo titulado "Taking facts seriously», en el que se argumentaba que el tema de la prueba y la determinación de los hechos requiere un lugar más destacado en el Derecho. El objetivo que se propone el autor en esta ocasión es el siguiente. En primer lugar, plantear nuevamente la tesis original y argumentar que merece ser atendida porque el estudio de la prueba es importante para entender el Derecho y para la práctica jurídica. En segundo lugar, examinar de qué manera se puede construir un curso sobre la prueba en condiciones de sobrecarga del plan de estudios y de desatención de las cuestiones probatorias en otras partes del mismo. En tercer lugar, mostrar algunos aspectos en los que el reciente surgimiento de la prueba como un ámbito multidisciplinario puede afectar el estudio y la enseñaza de la prueba en el Derecho.

Palabras clave: W. TWINING, prueba, enseñanza del Derecho, chart method, J. H. WIGMORE.

ABSTRACT. This essay is a sequel of a paper called "Taking facts seriously» in which the author argued that the subject of evidence, proof and fact-finding deserves a more salient place in the discipline of Law. The aim of this paper is threefold. First, to restate the original thesis and to argue that it deserves support because many of the issues involved are important to understanding and practicing law. Secondly, to consider how a coherent single introductory course on evidence can be constructed in conditions of curriculum overload and neglect of evidentiary issues in other parts of the curriculum. Thirdly, to suggest some ways in which the recent emergence of evidence as a multidisciplinary field in its own right might affect the study and teaching of evidence in law.

Keywords: W. Twining, evidence, proof, legal education, chart method, J. H. WiGMORE.

* Fecha de recepción: 28 de septiembre de 2009. Fecha de aceptación: 29 de octubre de 2009.

Una versión revisada de este trabajo fue publicada en 2005, en el Journal of Legal Education, 55 (3). Agradezco a los participantes en los congresos celebrados en University College London, en la Universidad de Illinois y en la Universidad de Nottingham por sus valiosos comentarios, y a P. ROBERTS por el estimulante intercambio de opiniones a lo largo de todo este tiempo. La presente traducción corresponde a la versión publicada en TwINING, 2006, cap. 14. 


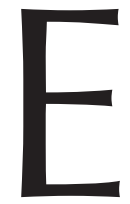

n 1980 escribí un artículo titulado «Los hechos en serio», el cual, si bien es bastante conocido, ha tenido poca repercusión ${ }^{1}$. Sigo pensando que la tesis allí expuesta es correcta y que es importante para nuestras ideas y para la práctica en el ámbito académico del Derecho, en relación con el método jurídico, la teoría del derecho, el derecho probatorio y la enseñanza del Derecho en general. La suerte que ha corrido me recuerda una frase de K. LLEWELLYN: «Cuando Cicerón pronunciaba un discurso, la gente decía: "Ningún mortal es tan elocuente”; cuando Demóstenes pronunciaba un discurso, la gente gritaba: “¡A LA CARGA!"». El fallo parece atribuible a la falta de una buena defensa.

La conferencia tuvo lugar con motivo de la inauguración de un nuevo edificio de la facultad de Derecho en la ciudad de Victoria, en la provincia de la Columbia Británica; el contexto era un simposio sobre la enseñanza del Derecho; el auditorio estaba conformado principalmente por jueces, juristas prácticos, juristas académicos y por estudiantes de Derecho. Probablemente no había presentes más de cuatro o cinco especialistas sobre la prueba. Mi tesis era que el tema de la prueba y la determinación de los hechos $(E P F)$ *, requiere un lugar más destacado en el Derecho. En el trabajo sostenía que la investigación y el manejo de los hechos, al igual que la argumentación sobre cuestiones de hecho controvertidas en los diferentes contextos jurídicos (no sólo en los tribunales), requieren tanta atención y tanta exigencia intelectual como las cuestiones de interpretación y argumentación sobre cuestiones de Derecho. De cara al público en general, se trataba de defender la importancia del estudio de los hechos en la enseñanza del Derecho.

El trabajo fue bien recibido cuando se publicó, pero no fue convincente, quizá por una cuestión del auditorio al que se pensó que estaba dirigido: a los juristas prácticos les gustó mucho, pero era sobre la enseñanza del Derecho; en el ámbito académico, muchos juristas percibieron que el artículo estaba dirigido a especialistas en temas de prueba y, por tanto, sin ninguna vinculación con sus intereses; algunos profesores de Evidence percibieron el trabajo como una crítica demoledora y poco diplomática a los cursos tradicionales de Derecho probatorio; mientras que otros lo vieron como una mala promoción de los diagramas de WigMORE.

En esta ocasión, el público al que me dirijo está conformado en términos generales por especialistas en el tema de la prueba y por juristas académicos. El objetivo que persigo es triple. En primer lugar, reformular la tesis original y argumentar que merece ser atendida, no simplemente o únicamente para mitigar presiones en el plan de estudios, sino porque muchas de las cuestiones son importantes para entender el Derecho y la práctica jurídica. En segundo lugar, examinar de qué manera se puede construir un curso sobre la prueba en condiciones de sobrecarga del plan de estudios y de desatención de las cuestiones probatorias en otras partes del mismo ${ }^{2}$. En tercer

1 Vid. TwinING, 2006, cap. 2. Este artículo fue publicado originalmente en GOLD, 1982 y en 1988, Journal of Legal Education, 34 (22).

* El autor utiliza a lo largo del trabajo la abreviación EPF para referirse a Evidence Proof and Factfinding, esto es, a las cuestiones referidas a los elementos probatorios o pruebas (evidence), al proceso de prueba (proof) y a la determinación de los hechos. En la presente traducción se ha optado por traducir esta abreviación como «prueba y determinación de los hechos», en el entendido de que «prueba» hace referencia en este contexto tanto a las pruebas (evidence) como al proceso de prueba (proof) [N. del T.].

2 Este artículo se ocupa del tema de la prueba en los programas de Derecho en los países del Common Law. El argumento general es aplicable tanto a los programas de licenciatura (como en Inglaterra) como a los 
lugar, mostrar algunos aspectos en los que el reciente surgimiento de la prueba como un ámbito multidisciplinario puede afectar al estudio y a la enseñaza de la prueba en el Derecho.

\section{EL TEMA DE LA PRUEBA AMERITA UN LUGAR MÁS DESTACADO EN EL DERECHO}

En lugar de volver a examinar los temas tratados en el artículo anterior, voy a reformular brevemente las razones por las que considero que el tema de la prueba es importante.

\subsection{Entender el tema de la prueba es una parte importante de lo que implica entender el Derecho}

La prueba es importante para la teoría del Derecho porque plantea una gran variedad de cuestiones teóricas que por lo general no están incluidas en la agenda de la mayoría de los teóricos del Derecho. La práctica consistente en tratar el «razonamiento jurídico» como si estuviera relacionado únicamente con cuestiones de Derecho tradicionalmente consideradas como difíciles es sintomático de distorsiones aún más fundamentales en la agenda de la teoría del Derecho, como he tenido ocasión de mostrar en otro lugar ${ }^{3}$.

Llama la atención que las cuestiones relativas a la determinación de los hechos raramente ocupan una parte importante en las teorías de la decisión judicial ${ }^{4}$. ¿Es concebible una teoría coherente e integral de la decisión judicial que ignore decisiones sobre las cuestiones de hecho, sobre el procedimiento y sobre la resolución? ¿Cómo se puede justificar la separación entre dos conjuntos de literatura a partir de la problemática distinción entre cuestiones de hecho y cuestiones de Derecho? ${ }^{5}$.

Resulta extraño que conceptos probatorios básicos como el de relevancia, materialidad, fuerza probatoria, probabilidad, credibilidad y presunción hayan recibido tan poca atención por parte de la teoría analítica del derecho ${ }^{6}$.

Llama la atención que la mayoría de las discusiones sobre el razonamiento y la interpretación jurídica se enfoquen en cuestiones de Derecho, con escasa o sin ninguna referencia ya no sólo a las cuestiones de hecho, sino de manera más general, al razonamiento y a la racionalidad en otros contextos jurídicos, tales como la investigación, la negociación y la fijación de la sanción. Las relaciones entre los distintos tipos de razonamientos de los juristas no han sido suficientemente exploradas.

programas de posgrado (como en los Estados Unidos y en otros países del Common Law). No obstante, se tiene en cuenta el hecho de que las presiones en el plan de estudios, ya sea por parte de los exámenes profesionales, por falta de tiempo o por otros factores, varían entre las distintas jurisdicciones.

3 Twining, 1997, cap. 6, y Twining, 2006: cap. 8.

4 Por ejemplo, la teoría moderna más importante de la decisión judicial, como la que desarrolla DwORKIN, 1977 y 1986, ignora casi por completo cuestiones tales como la determinación de los hechos y el establecimiento de la sanción.

5 Cfr. Allen y Pardo, 2003, y Kirgis, 2004.

6 Una excepción clásica es MONTROSE, 1984. El argumento a favor de una concepción y una agenda más amplia de la teoría analítica del Derecho es desarrollado en TwINING, 2005. 
Es extraño que no se perciba que las cuestiones acerca de la relación entre las narraciones y los argumentos, entre «holismo» y «atomismo» y las cuestiones de coherencia son centrales en las teorías del razonamiento y de la racionalidad jurídica ${ }^{7}$. El papel de la narración en el discurso jurídico y las cuestiones acerca de la relación entre la narración, el razonamiento, la argumentación y la persuasión se distorsionan si la narración y los relatos se examinan únicamente en relación con cuestiones de hecho controvertidas en la decisión judicial ${ }^{8}$. Los relatos y la narración de relatos también son importantes en la investigación, en la mediación, en la negociación, en la apelación, en la sentencia y en las predicciones de peligrosidad, por ejemplo ${ }^{9}$. Una teoría general de la narración en el Derecho y en la argumentación jurídica necesita abarcar todas estas cuestiones. Algunos de estos temas han sido discutidos de manera ecléctica bajo el título de «Derecho y literatura» ${ }^{10}$, pero hace falta desarrollar un esquema integral en el que se puedan examinar y relacionar mutuamente todas estas líneas de investigación.

La teoría del Derecho necesita tomar más en serio las cuestiones relacionadas con la prueba en una concepción equilibrada de lo que implica entender el Derecho ${ }^{11}$. La desatención de estas cuestiones conduce a una imagen distorsionada de la decisión judicial, del litigio, del razonamiento jurídico y de la práctica jurídica.

\subsection{La prueba debería jugar una función importante en el estudio de muchas áreas especializadas del Derecho}

Las cuestiones de hecho y otras cuestiones probatorias se presentan en todos los ámbitos del Derecho. Algunas de estas cuestiones se presentan, por ejemplo, en temas como la discriminación, la disputa por tierras y la violación ${ }^{12}$. Este es un punto ampliamente aceptado, pero que es atendido de manera desigual. También se presentan problemas de solapamiento entre los cursos sobre la prueba y el procedimiento, en instituciones jurídicas (como el jurado o el interrogatorio policial, por ejemplo) y en los procesos jurídicos. Por otra parte, hay una literatura creciente sobre los problemas de

7 El ensayo de N. MACCORMick, 1984, ha estimulado una extensa literatura, pero principalmente en relación con la interpretación y aplicación de las reglas jurídicas. Vid., recientemente, MACCORMICK, 2005, caps. 10 y 11, y PARDO, 2000.

8 Twining, 2006, cap. 10.

9 Twining, 2006, cap. 10. Vid., además, SCHum, 1999/2001; Binder y BERGMAn (investigación de hechos), 1984, LLEWELLYN, 1960 (casos de apelación); SHAPLAND, 1981 (alegaciones de reducción o atenuación), BECHER-MONAS, 2003 (predicciones de peligrosidad). En la nueva edición de Analysis of Evidence se adopta un modelo que abarca cualquier tipo de proceso y se hace hincapié en la investigación de los hechos (cap. 2) y en los estándares de evaluación de las pruebas en distintos tipos de decisión (cap. 8).

10 Por ejemplo, LEDWON, 2003.

11 P. ROBERTS señala que «existe la sospecha de que, siendo un jurista, TwINING esté tratando de convertir el Derecho probatorio en una rama de la teoría del Derecho». ROBERTS, 2002. Esto es una interpretación errónea. Es posible que sea un imperialista en cuestiones probatorias, pero mi principal argumento es que la falta de atención a estas cuestiones por parte de la teoría del Derecho tiende a promover un entendimiento distorsionado del Derecho y de los fenómenos jurídicos. Como discípulos relativamente fieles de K. LEWELLYN, mi coautor, T. ANDERSON y yo rechazamos distinciones tajantes entre «teoría» y «práctica», pero como pone de manifiesto RoBERTS, nuestra enseñanza del «Análisis de las Pruebas» pretende ser mucho más práctica que el estudio formal del Derecho probatorio.

12 Por ejemplo, Ellison, 1998, y KibBle, 2004. 
prueba que se presentan en los tribunales penales internacionales (no sólo a propósito de las reglas de prueba, sino respecto a si los crímenes contra la humanidad plantean alguna cuestión especial relacionada con la prueba) ${ }^{13}$. Algunas de estas cuestiones se vinculan con preguntas generales acerca de hasta qué punto los principios de la prueba son independientes del contexto e independientes del contenido ${ }^{14}$. Estas cuestiones no sólo se presentan en contextos jurídicos, sino de manera más general y pronunciada, cuando se examina la prueba desde perspectivas multidisciplinarias ${ }^{15}$.

\subsection{La prueba es importante para la práctica jurídica}

Cuando J. FRANK sostuvo que más del 90 por 100 de las decisiones judiciales y las diligencias previas al juicio estaban más relacionadas con dudas e incertidumbres sobre los hechos que sobre cuestiones controvertidas de Derecho, en cierto modo estaba subestimando el problema, porque sólo estaba interesado en el litigio y se enfocaba principalmente en el juicio con jurado ${ }^{16}$. Sin embargo, el punto central de su argumento era fundamentalmente correcto, a saber, que el razonamiento inferencial y otros aspectos del manejo de la información son importantes en la mayoría de contextos de la práctica jurídica y que han sido relativamente desatendidos en la enseñanza del Derecho y en la formación jurídica. El énfasis que se hace en las Federal Rules en los exámenes de habilitación y en las reglas de prueba en la formación policial, pasando por alto el razonamiento inferencial, son algunos de los principales ejemplos ${ }^{17}$.

\subsection{El tema de la prueba (EPF) es una herramienta adecuada para desarrollar algunas habilidades intelectuales básicas de carácter general}

El desarrollo de habilidades relacionadas con el razonamiento inferencial forma parte del método jurídico y es tan demandante como las cuestiones de Derecho ${ }^{18}$. En el ámbito de la prueba y la determinación de los hechos $(E P F)$, los principales ingredientes consisten en: i) manejar datos complejos y construir y examinar de manera crítica argumentos complejos, incluyendo técnicas para organizar pruebas, el uso de tablas cronológicas; clasificar pruebas en función de la fuente de la que provienen; diagramas de WIGMORE y relatos ${ }^{19}$; habilidades de razonamiento inferencial y análisis microscópico; ii) algunas habilidades matemáticas básicas ${ }^{20}$; iii) construir, transmitir y rebatir historias persuasivas, y iv) cuestiones éticas relacionadas con

\footnotetext{
13 Vid., más abajo, n. 28.

14 Vid. el trabajo de D. Schum en Twining y HAmpSHER-Monk (eds.), 2003: cap. 1, y Twining, 2006, cap. 15

15 Véase Twining, 2006, pp. 428-429.

16 Sobre Frank, vid. TwINING, 2006, pp. 116-119.

17 LEARY, 2004, cap. 1.

18 Sobre la respuesta a las objeciones de que las «habilidades» intelectuales no son propias de una educación de carácter liberal, vid. TwINING, 1997, cap. 9.

19 En Anderson, Schum y TwINING, 2005, caps. 4-6, se discuten ampliamente los diferentes métodos para ordenar las pruebas y para estructurar argumentos.

20 Vid. Anderson, Schum y Twining, 2005, Appendix I, Hawkins y Hawkins, 1998, y Wagenaar y Crombag, 2005.
} 
todos estos aspectos. Algunos profesores tratamos de desarrollar estas habilidades en cursos sobre la prueba ${ }^{21}$. Esto puede funcionar bastante bien, pero supone dos costos. En primer lugar, algunos de estos temas son importantes en otros contextos. Aislar estos temas en contextos en los que la atención se centra en el manejo de los hechos los separa de manera artificial, tanto desde un punto de vista teórico como práctico, de otros contextos relevantes. Las habilidades matemáticas, por ejemplo, no sólo se aplican en la probabilidad y en la prueba ${ }^{22}$. En segundo lugar, si no se abordan estas cuestiones de manera adecuada, se ejerce una mayor presión en los cursos sobre la prueba.

\subsection{A medida que el Derecho se vuelve más cosmopolita, se presentan nuevas e interesantes cuestiones de comparación, generalización e hibridación en relación con la prueba y la determinación de los hechos}

En los últimos años se ha prestado una atención cada vez mayor al Derecho procesal y a la prueba desde una perspectiva comparada, así como a las implicaciones de la globalización para el estudio del Derecho. El trabajo pionero de M. DAMASKA, por ejemplo, ha proporcionado un modelo apto para comparar no sólo sistemas procesales, sino también la manera en que las cuestiones probatorias son examinadas en dichos sistemas ${ }^{23}$. H. CROMBAG, P. vON KOPPEN y W. WAGENAAR han aplicado a casos holandeses ideas e hipótesis desarrolladas por psicólogos y gente de otros ámbitos en los Estados Unidos ${ }^{24}$. Este trabajo plantea interrogantes acerca de hasta qué punto «viajan bien» los principios lógicos, los conceptos probatorios, las investigaciones empíricas y doctrinas concretas a través de las distintas tradiciones y culturas jurídicas ${ }^{25}$. Tengo noticia por parte de los juristas continentales de que «la Tradición Racionalista» parece encajar mejor en los sistemas de Derecho continental que en los del Common Law $^{26}$. Las Comisiones de la Verdad y de Reconciliación, los tribunales penales internacionales y el arbitraje comercial internacional plantean cuestiones interesantes sobre la prueba en estos contextos específicos ${ }^{27}$. Del mismo modo, es evidente que la Convención Europea de Derechos Humanos ha tenido una influencia tanto directa como indirecta en el Derecho probatorio del Reino Unido ${ }^{28}$.

21 Si se considera que las historias desempeñan una función importante en la determinación de los hechos, pero que al mismo tiempo pueden ser utilizadas para engañar, entonces la enseñanza de habilidades para contar relatos persuasivos plantea algunas dificultades éticas análogas a los problemas clásicos de la enseñanza de la retórica.

22 Vid., por ejemplo, la variedad de temas abordados en FinKELSTEIN y LEVIN, 2001; GASTWIRTH (ed.), 2000, y DAWID, 2005.

23 DAMASKA, 1986 y 1997.

24 Wagenaar, Von Koppen y Crombag, 1993 (analizado en Twining, 2002, cap. 13); Van Kampen, 1998; Anderson, 1999; Malsch y NijBoer, 1999; WaganaAr y Crombag, 2005.

25 Sobre la idea de que los conceptos «viajan bien», vid. TwINING, 2005.

26 Vid. Twining, 2006, pp. 85-86.

27 Vid., por ejemplo, las cuestiones sumamente diversas planteadas por KROG, 1999 (sobre la Comisión de la Verdad y Reconciliación en Sudáfrica); COGAN, 2000 (sobre las pruebas en tribunales penales internacionales); MAY et al., 2001, y WALD, 2003 (sobre el Tribunal Penal Internacional para la antigua Yugoslavia); HIRSCH, 2003 (sobre juicios por genocidio); DEZALAY y GARTH, 1996 (arbitraje internacional), y ROEBUCK, 2001.

28 Vid., por ejemplo, la Tabla de Instrumentos Internacionales y Legislación Comparada en ROBERTS y ZuCKERMAN, 2004; cfr. SEDLEY, 2005: 7: «No concibo la manera en que hoy en día se enseñe Derecho probato- 
El tema de la prueba se presenta de este modo como un ámbito claramente multidisciplinario. Este es quizás el desarrollo más importante de los últimos años y será discutido en la última sección.

\subsection{La enseñanza de la prueba a nivel universitario: sugerencia para un modelo ${ }^{29}$}

La tesis de que la prueba y la determinación de los hechos $(E P F)$ ameritan un lugar más destacado en la disciplina del Derecho supone que este tema requiere un mayor espacio en los planes de estudio de los programas de licenciatura y posgrado. Del mismo modo, debería darse una mayor atención a las cuestiones probatorias en las materias de metodología jurídica, teoría del Derecho, sistemas jurídicos o Derecho comparado y otras materias similares, o bien debería haber espacio para al menos dos cursos en la parte general. Sin embargo, hasta que esta tesis cobre aceptación, tenemos que afrontar el problema de diseñar cursos coherentes sobre la prueba en un contexto de sobrecarga de los planes de estudio.

En un interesante artículo titulado «Rethinking the Law of Evidence» ${ }^{30}$, P. ROBERTS suscribe mi tesis, pero critica la defensa que hago de ella. ROBERTS se enfoca en la enseñanza de cursos sobre la prueba en licenciatura, los cuales son los más problemáticos en términos de sobrecarga. A la par de que es cuidadoso al reafirmar su compromiso con el pluralismo en la enseñanza del Derecho a nivel universitario, ROBERTS construye un modelo para un curso de licenciatura sobre la prueba que se diferencia de otros cursos más específicos de Derecho probatorio ${ }^{31}$.

ROBERTS expone un principio básico para la enseñanza de la prueba basado en una visión tradicional del grado de Derecho como la última etapa formal de una educación liberal ${ }^{32}$. A su juicio, la carrera de Derecho puede contribuir a este objetivo «inculcando habilidades generales relevantes, sólidas y (más o menos) adaptables, contribuyendo a que los estudiantes continúen aprendiendo de por vida y a que promuevan una ciudadanía responsable» ${ }^{33}$.

Vamos a aceptar lo anterior como punto de partida para elaborar un curso de licenciatura sobre la prueba ${ }^{34}$. Los cimientos de la concepción de ROBERTS de un curso

rio, por ejemplo, sin incorporar la jurisprudencia de la [Convención Europea de Derechos Humanos]; CHOO y NASH, 2003, y JACKSON, 2005.

29 NiCOLSON, 1997, y MURPHY, 2001, concuerdan con el enfoque general, pero sugieren estrategias distintas. $C f r$. MACK, 1998, donde se analiza el tema de la diversidad como una cuestión implícita en las cuestiones probatorias de relevancia y de la lógica de la prueba.

30 ROBERTS, 2002.

31 ROBERTS y ZUCKERMAN, 2004.

32 Una formulación clásica de esta perspectiva puede verse en el artículo de K. LLEWELLYN, «The Study of Law as a Liberal Art», reimpreso en LLEWELLYN, 1962, cap. 17.

33 LLEWELLYN, 1962, 301.

34 El trabajo de RoBERTS está enfocado únicamente a Inglaterra y Gales. Paradójicamente, uno esperaría que las facultades de Derecho en Estados Unidos tuvieran una orientación más profesional. Sin embargo, el principal obstáculo para desarrollar habilidades intelectuales de análisis y argumentación sobre cuestiones de hecho son los exámenes de habilitación (bar examinations). En estos exámenes se da un mayor énfasis al conocimiento detallado de las Federal Rules of Evidence y a la capacidad de aplicarlas que a las habilidades para analizar, ordenar y construir argumentos basados en conjuntos de pruebas de distintos tipos. De este modo, 
sobre la prueba (suponiendo que no haya otra parte en el programa de estudios en el que se aborden a fondo las cuestiones probatorias) son los siguientes ${ }^{35}$ :

1. El estudio del derecho probatorio debería situarse en su contexto procedimental, institucional y teórico ${ }^{36}$.

2. El estudio de la prueba debe abarcar todas las etapas del proceso jurídico y no debería limitarse a los juicios ante tribunales, ni a los juicios con jurado. En concreto, hay una mayor necesidad que en el pasado de enfocarse en los aspectos previos del proceso (incluyendo la investigación de hechos). En resumen, el contexto procedimental supone un modelo de litigio que abarque cualquier tipo de proceso.

3. El contexto teórico incluye cuestiones sobre epistemología, razonamiento inferencial y principios de moralidad política (incluyendo feminismo ${ }^{37}$, análisis económico $^{38}$, teoría crítica ${ }^{39}$ y postmodernismo ${ }^{40}$ ).

4. Las relaciones entre el análisis de hechos y las reglas de prueba necesitan ser clarificadas y tratadas como partes de un todo coherente ${ }^{41}$.

5. El tema de la relevancia es el principal vínculo entre el análisis de hechos y la doctrina jurídica:

a) La relevancia es el mecanismo de exclusión más importante.

b) Entender la relevancia supone entender los principios y las características del razonamiento inferencial.

c) Los principios de prueba son previos a las demás reglas de exclusión, porque estas últimas se ocupan de la exclusión de pruebas relevantes.

d) El Derecho probatorio puede ser considerado como un conjunto coherente a partir de los principios de inclusión y exclusión de THAYER, los cuales son formulados en términos de relevancia dentro del esquema básico de la argumentación ${ }^{42}$.

6. La lógica de la prueba es un elemento importante para estudiar conceptos básicos del Derecho probatorio como por ejemplo, el testimonio de referencia (bearsay), los hechos notorios (judicial notice), la prueba de hechos similares (similar fact evidence), el prejuicio indebido (prejudicial effect) y el valor probatorio, las cargas y los estándares de prueba (al igual que estándares para otro tipo de decisiones) ${ }^{43}$ y las presunciones ${ }^{44}$.

\footnotetext{
como ocurre comúnmente, una filosofía de la enseñanza de carácter liberal puede estar en una mejor posición para contribuir al desarrollo de habilidades prácticas transferibles que sean útiles.

35 Hago esta formulación en mis propios términos y tratando de presentar las ideas de una manera más concreta, pero en términos que, a mi modo de ver, ROBERTS consideraría aceptables.

36 Roberts y Zuckerman, 2004, Prólogo. Prácticamente todas estas ideas son analizadas en Twining, 2006.

37 Por ejemplo, ChILDS y ELLISON (eds.), 2000.

38 POSNER, 1999, y LEMPERT, 2001.

39 Siegel, 1994, y NiCOLSON, 1994.

40 NiCOLSON, 1994. Sobre mi opinión acerca de la epistemología posmodernista, vid. TwinING, 2002, pp. 289-309. Sobre los derechos humanos, vid. supra n. 29.

41 Vid. Anderson, Schum y Twining, 2005, cap. 11; Acorn, 1991, Roberts y Zuckerman, 2004, passim.

42 THAYER, 1898; analizado en TWINING, 2006: cap. 6.

${ }^{43}$ Sobre el amplio concepto de «estándares de decisión» vid. ANDERSON, SCHUm y Twining, 2005, cap. 5.

${ }^{44}$ ANDERSON, SCHUM y TwINING, 2005, cap. 11 y passim. Al argumentar que se debe prestar más atención al tema de la relevancia, ROBERTS sugiere que tras un estudio preliminar a comienzos del curso, se puede refor-
} 
7. El tema cada vez más importante de la prueba científica y la prueba pericial (expert evidence) es un buen punto de referencia para abordar cuestiones epistemológicas centrales sobre la prueba ${ }^{45}$.

8. Las cuestiones probatorias se presentan en cualquier tipo de procedimiento, en todos los procesos jurídicos y en cada etapa. Sin embargo, el tema resulta más manejable y coherente si nos enfocamos sólo en un tipo de procedimiento - a saber, en el proceso penal, el cual, en la práctica, se ha convertido en el principal foco de atención de los cursos sobre la prueba en Inglaterra ${ }^{46}$.

9. A pesar de (8), los principios básicos del razonamiento y del análisis sobre cuestiones de hecho y conceptos básicos como relevancia, credibilidad y fuerza probatoria, son independientes del contenido (SCHUM) e independientes del contexto, dado que no sólo trascienden la división penal/civil, sino que viajan bien a través de las tradiciones jurídicas occidentales e incluso a través de barreras disciplinarias ${ }^{47}$.

10. A medida que el Derecho se vuelve más cosmopolita, inevitablemente se va a tener que prestar más atención a los aspectos de la prueba en el ámbito europeo, internacional, trasnacional y comparativo.

11. Un curso sobre la prueba puede ser un vehículo para desarrollar habilidades matemáticas relacionadas con el Derecho.

12. Las narraciones basadas en pruebas desempeñan un papel central en la construcción y presentación de argumentos sobre cuestiones de hecho ${ }^{48}$.

ROBERTS y yo estamos de acuerdo en estas doce proposiciones. Sin embargo, me gustaría desarrollar algunos puntos generales que pueden sacar a la luz algunas diferencias, al menos de énfasis y de prioridades.

zar su explicación al relacionarlo con otros temas, como la prueba de una mala conducta previa, el testimonio de referencia o el silencio del acusado (pp. 306-307). Concuerdo con ROBERTS en este punto. Vid., adicionalmente, ROBERTS y ZUCKERMAN, 2004, passim.

${ }^{45}$ Anderson, Schum y Twining, 2005, cap. 5 y passim. Roberts, 2002, pp. 324-328. Las relaciones entre la epistemología y la prueba científica han sido exploradas de manera sobresaliente por la filósofa S. HAACK. Vid., especialmente, HAACK, 2003a, cap. 9; HAACK, 2003b, y HAACK, 2004. Vid. asimismo, BREWER, 1998. Respecto a las implicaciones de los avances en la ciencia y en la tecnología vid. TwinING, 2006, p. 230, n. 35.

46 Concuerdo con la idea de que es deseable integrar el derecho probatorio civil y penal y vincularlo con sus específicos contextos procedimentales. Sin embargo, vale la pena hacer hincapié en que la lógica de la prueba puede aplicarse a otros contextos, al igual que algunos conceptos básicos como el de relevancia, credibilidad y fuerza probatoria (cfr. ROBERTS, 2002, p. 317). En la nueva edición de Analysis of Evidence (2005) se incluyen ejercicios de casos penales y civiles, ejemplos de análisis de inteligencia y eventos históricos a fin de destacar esta transferibilidad.

47 Sobre la idea de cruzar fronteras disciplinarias, vid. SCHum, 1994 y 2001, y «la hipótesis de Twining» en Twining y HAMPSHER-MONK, 2003, analizados en Twining, 2006, cap. 13. Sobre la idea de trascender culturas jurídicas, vid. Twining, 2006, n. 22. Para un intento de aplicar una versión modificada del análisis wigmoreano a un problema histórico (la fecha de desaparición de la escritura cuneiforme), véase el trabajo de T. ANDERSON y M. GELLER titulado «The Last Wedge» en TwinIng y HAMPSHER-Monk, 2003, caps. 3-5.

${ }^{48} \mathrm{La}$ noción de «narraciones basadas en pruebas» es similar a la noción de «narraciones ancladas» desarrollada por WAGENAAR, VON KROPPEN y CROMBAG, 1993, con la salvedad de que ellos otorgan un énfasis mucho mayor a las generalizaciones que a las pruebas concretas como «anclas» de las historias. Vid. TwINING, 2002, pp. 428-431. 


\subsubsection{La teoría de la prueba es importante para proporcionar un esquema coherente para el estudio del la prueba en el Derecho (EPF)}

Wigmore dividía el tema de la prueba en contextos jurídicos en dos partes complementarias: los «Principios de la Prueba» («proporcionados por la lógica, la psicología y la experiencia general») y las «Reglas del Juicio». WigmORE sostenía que los principios de la prueba eran anteriores a las reglas del juicio, que eran más importantes en la práctica y que habían sido desatendidos por los académicos y por los profesores de evidence. Esta desatención continuó hasta el surgimiento de lo que se conoce como «the New Evidence Scholarship» en los años setenta y ochenta, el cual hasta hoy no parece que haya estado acompañado por un desarrollo comparable de una «Nueva Enseñanza de la Prueba». Una de las razones de ello es la falta de un esquema coherente.

Si aceptamos que el estudio de la prueba se lleva a cabo de una manera más adecuada en el contexto de un modelo de litigio que abarque cualquier tipo de proceso (civil, penal o cualquier otro), el enfoque de WIGMORE en los juicios con jurado y su concepto de las «Reglas del Juicio» es demasiado restringido. No obstante, su concepción de la prueba en el Derecho como consistente en dos partes estrechamente vinculadas es digna de tomarse en cuenta. Yo prefiero hacer referencia a estas dos partes como los principios de la prueba (o la lógica de la prueba) y el Derecho probatorio, en lugar de subsumirlas dentro de una concepción amplia del Derecho probatorio. Pero esta es una mera cuestión de etiquetas ${ }^{49}$.

Para construir un esquema coherente del estudio de la prueba en el Derecho se requieren dos cosas más: una imagen coherente de cada uno de estos componentes y una concepción coherente de la relación entre estas dos partes.

A mi juicio, el modelo de «la Tradición Racionalista de los Estudios sobre la Prueba» proporciona la imagen más coherente de los principios de la prueba. Esta imagen puede resumirse en la proposición benthamita de que el objetivo primario del proceso jurídico consiste en la búsqueda de justicia por medio del Derecho, mediante la obtención de decisiones correctas a través de medios racionales ${ }^{50}$. No es necesario suscribir todos los elementos de este modelo ideal para utilizarlo como una herramienta organizativa; de hecho, mi formulación del modelo racionalista estaba diseñada específicamente para identificar puntos potencialmente importantes que pudieran ser cuestionados por las distintas clases de escépticos o por otros. Más bien, el modelo es sólido por dos razones: primero, porque representa un conjunto estable de presupuestos que han sido ampliamente compartidos por los principales autores que han escrito sobre el Derecho probatorio a lo largo de dos siglos en la tradición del Common Law. Estos presupuestos también sustentan importantes inten-

49 Sin embargo, hay buenas razones para optar por esta elección: el término «Derecho probatorio» tiene una asociación muy fuerte con las reglas de exclusión y a menudo conduce al presupuesto falaz de que el tema de la prueba en el Derecho es co-extensivo con las reglas de prueba. Esto lleva, en cambio, a exagerar la importancia de las reglas (TWINING, 2006, pp. 210-218) y a prestar poca atención a aspectos que no están regidos por reglas formales, como la relevancia, el peso y la argumentación. Vid. además, TWinING, 2006, 440.

50 Vid. Twining, 2006, cap. 3. 
tos de racionalizar el Derecho probatorio, incluyendo las Federal Rules of Evidence en los Estados Unidos. En segundo lugar, este modelo ideal sitúa desde el inicio el estudio de la prueba en su contexto ideológico, esto es, en relación con los valores subyacentes involucrados en el diseño y operación de los procedimientos jurídicos. En tanto que los principales valores que involucra — verdad, razón, justicia e interés público- son generalmente, y quizá esencialmente, controvertidos, lejos de ser un modelo funcionalista ingenuo y dogmático, proporciona un contexto para examinar cuestiones controversiales.

Históricamente, la mayor parte de las controversias que han tenido lugar en los estudios sobre la prueba en el ámbito angloamericano pueden ser ubicadas al interior del modelo racionalista, dado que se relacionan con prioridades entre la verdad y otros valores o porque representan distintas perspectivas de racionalidad (por ejemplo, baconianos frente a pascalianos), o diferentes teorías epistemológicas (por ejemplo, teorías de la verdad como correspondencia frente a teorías de la verdad como coherencia) o entre prioridades políticas (por ejemplo, valores del proceso o debido proceso frente al control social). Estas discusiones pueden verse como debates al interior de la tradición racionalista. Sin embargo, el modelo identifica un número de potenciales puntos de partida de uno o más presupuestos de dicha tradición, por ejemplo, cuestionamientos de filósofos escépticos, relativistas culturales, posmodernistas, o aquellos que dudan de la conveniencia, la viabilidad o del sentido de que el litigio se vincule centralmente con la verdad, la justicia o la razón ${ }^{51}$.

El modelo racionalista proporciona un punto de partida para examinar el tema de la prueba en el Derecho $(E P F)$ como un todo coherente, incluyendo poner en contexto la lógica de la prueba, la cual, en la tradición del Common Law, tiene sus raíces en la tradición empirista de Sir F. Bacon, J. Bentham, J. Stuart Mill, S. Jevons y A. Sidgwick, continuando con J. Cohen, S. Toulmin, D. Walton y D. Schum.

$¿$ Es posible dotar al Derecho probatorio de una estructura coherente a fin de que no sea visto solamente como un conjunto fragmentado y confuso de temas poco relacionados? I. Dennis, A. ZuCKerman, A. Ashworth y otros autores han explicado el Derecho probatorio sobre la base de sus principios subyacentes ${ }^{52}$; a pesar de ello, todavía sigo encontrándome con estudiantes en Estados Unidos y en Londres que tienen la impresión de que el Derecho probatorio consiste en un conjunto arbitrario de reglas técnicas. Esto sucede incluso con aquellos que han estudiado las Federal Rules, las cuales tienen una estructura claramente discernible, e incluso con los estudiantes que han estado expuestos al revoltijo y a la poca sistematicidad de la legislación y del case law en Inglaterra. La explicación de esta triste situación parece residir, a mi modo de ver, en que no se les ha presentado a los estudiantes un panorama coherente del Derecho probatorio y a que no han recibido una explicación adecuada del tema de la relevancia $^{53}$. Esto se debe posiblemente a que la relevancia no es una cuestión de Derecho y

51 Twining, 2006, cap. 4. Sobre el posmodernismo vid. Twining, 2002. Cfr. el interesante artículo de RISINGER: 2004 (argumentando a favor de que la especial posición moral de la idea de «inocencia de hecho» sea reconocida al interior de la Tradición Racionalista).

52 ZuCKERMAN, 1989; ASHWORTH, 1998; DENNIS, 2002; ROBERTS y ZuCKERMAN, 2004.

53 Cfr. Roberts, 2002, 306: «...relevancia es uno de los conceptos menos explorados y del que se abusa con mayor frecuencia en el Derecho probatorio». 
que para entender la relevancia se tiene que entender la lógica que presupone. Como plantea THAYER, «el Derecho no tiene ninguna jurisdicción sobre la lógica» ${ }^{54}$.

Es indudable que existen múltiples maneras de presentar las reglas de la prueba en un esquema coherente. Mi punto de vista, el cual es bastante aceptado, es que nuestro Derecho probatorio está basado en la teoría thayeriana de que las reglas de la prueba son una serie de excepciones dispares al principio de prueba libre, donde prueba libre hace referencia a los principios tradicionales del razonamiento inferencial práctico ${ }^{55}$. Es necesario entender el principio antes de estudiar las excepciones, lo que supone entender la lógica de la prueba. Es posible que THAYER haya exagerado la importancia del jurado en el desarrollo histórico del derecho probatorio y que haya adoptado una visión limitada del Derecho probatorio al equipararlo con las reglas de exclusión en el proceso (aunque sí escribió sobre las presunciones, las cargas de la prueba y los hechos notorios). Sin embargo, THAYER acertó completamente al sostener que las reglas de la prueba necesitan ser concebidas dentro del esquema de la argumentación.

Desde un punto de vista pedagógico, el tema de la relevancia es el vínculo indiscutible entre la lógica de la prueba y las reglas de la prueba ${ }^{56}$. Comprender la relevancia supone comprender la lógica de la prueba en un esquema en el que se aborden los principios de la prueba y las reglas de la prueba como pertenecientes a una materia coherente. Estoy de acuerdo con ROBERTS en que la relevancia no debería verse como un tema que baste abordar en la primera semana de clase ${ }^{57}$. Por el contrario, debe ser un tema recurrente al estudiar el resto de los temas del Derecho probatorio. En el siguiente epígrafe voy más allá y argumentaré que comprender la lógica de la prueba involucra tanto la adquisición de conocimientos como el desarrollo de habilidades. El estudio de este tema de manera directa y desde el comienzo del curso, reforzando los conceptos y habilidades a lo largo del curso, es una manera más rápida y efectiva de entender la prueba. Más aún, sirve para desarrollar importantes habilidades generales de carácter intelectual.

\subsubsection{Estudiar qué y aprender cómo}

Es necesario establecer una clara distinción entre aprender sobre el razonamiento y aprender cómo razonar. Las habilidades básicas del razonamiento inferencial sobre cuestiones de hecho forman parte del «método jurídico» o de lo que se considera que implica «pensar como un abogado» al igual que las habilidades básicas para construir y examinar críticamente argumentos sobre cuestiones de Derecho.

Una cosa es describir, interpretar y analizar el razonamiento de los jueces o de los abogados de las partes; otra muy distinta es dominar las habilidades relacionadas con la construcción de argumentos válidos, convincentes y adecuados sobre cuestiones de Derecho. Describir al juez Hércules es una cosa, emularlo es una cuestión distinta. La mayor parte de la enseñanza y la literatura versan sobre el «razonamiento jurídico».

\footnotetext{
54 THAYER, 1898, 314n.

55 Twining, 2006, cap. 6.

56 ROBERTS: 2002, pp. 306-307.

57 Ibid.
} 
En algunos libros y cursos sobre el «método jurídico» se desdibuja esta distinción ${ }^{58}$. Consideraciones análogas son aplicables al razonamiento sobre cuestiones de hecho. El libro de ROBERTS y ZuCKERMAN titulado Criminal Evidence es el último de una serie de textos sobre la prueba que se sitúan en la dirección de reconocer expresamente que la lógica de la prueba (o el análisis de los hechos) es una parte integral de la comprensión del tema de la prueba en el Derecho. No obstante, son pocos los autores que van más allá de explicar los distintos tipos de razonamiento que están presentes. Por ejemplo, bajo el título de «tomar los hechos en serio» ROBERTS y ZUCKERMAN dedican más de veinte páginas a conceptos básicos del razonamiento inferencial, al tema de la probabilidad y a discusiones sobre el teorema de Bayes en contextos jurídicos. Una cosa es tener en cuenta esos debates, otra distinta es aprender cómo utilizar el teore$\mathrm{ma}^{59}$. Esta parte del libro claramente cae dentro de la categoría de «estudiar qué» más que «aprender cómo». Esto es comprensible dado el formato del libro de texto e incluso algunos profesores de evidence considerarían que es un avance significativo. Sin embargo, la cuestión es si es deseable y viable ir más allá de este punto para tratar de contribuir a que los estudiantes controlen las habilidades básicas del razonamiento.

P. ROBERTS reconoce expresamente que un curso sobre la prueba es un vehículo adecuado para «inculcar habilidades (más o menos) transferibles, relevantes, sólidas y adaptables» ${ }^{60}$. Yo sería más enfático que ROBERTS por dos razones. En primer lugar, el dominio de habilidades básicas (y, sólo a un nivel ligeramente menor, algunas habilidades matemáticas básicas) es una de las cosas más valiosas que puede ofrecer una buena enseñanza del Derecho de carácter liberal. El desarrollo de estas habilidades se consigue mejor a través del estudio directo de las mismas más que por ósmosis ${ }^{61}$. En segundo lugar, si los principios de la prueba, i. e. el razonamiento inferencial práctico tradicional, dan sustento al Derecho probatorio, la experiencia sugiere que ésta es un área en la que aprender cómo es la vía más rápida para el aprendizaje.

¿Si se considera deseable inculcar habilidades básicas del razonamiento inferencial, cuál es la mejor manera de llevarlo a cabo y cuánto tiempo hace falta? Lo que WiGMO$\mathrm{RE}$ denominaba «la lógica de la prueba» consiste en aplicar los principios generales de la lógica inferencial en contextos jurídicos. Una posibilidad puede ser exigir que los estudiantes tomen un curso de lógica ya sea como parte de la carrera o en un curso previo a su ingreso a la carrera de Derecho (lo cual es lo más común en los Estados Unidos). Otra posibilidad sería utilizar un libro tradicional de lógica o de pensamiento crítico («critical thinking»), complementándolo con algunos ejemplos jurídicos. Desafortunadamente, esto no funciona muy bien. En primer lugar, la mayoría de cursos y libros de lógica se ocupan de la lógica formal, con un mayor énfasis en la deducción, mientras

58 Una excepción es HANSON, 2003, donde se aborda de manera detallada el razonamiento sobre cuestiones de Derecho y de hecho. Sobre las conexiones con la práctica en los tribunales, vid. PALMER, 2003, y MAUET, 2005. J. Covington trata de combinar ambos enfoques en su reciente trabajo sobre la prueba: Covington, 2006.

59 Sobre bayesianos y «escépticos bayesianos» vid. ROBERTS y ZuCKERMAn, 2004, pp. 123-132. Los principales desacuerdos se dan en relación con las condiciones de aplicación del Teorema de Bayes más que respecto de su validez. Para una visión menos escéptica vid. DAWID, 2002, y ANDERSON, SCHUm y TwinING, 2005, Appendix 1.

60 RoberTs, 2002, p. 301.

${ }^{61}$ Twining, 1997, pp. 181-183. 
que generalmente se acepta que el razonamiento jurídico es básicamente informal ${ }^{62}$. El segundo punto y probablemente el más importante es que una de las habilidades más importantes en el Derecho se relaciona con la construcción de argumentos complejos - lo que indistintamente se conoce como «manejo de hechos» $\mathrm{u}$ «ordenación de pruebas» («evidence marshaling»). Prácticamente todos los manuales y cursos de lógica se enfocan en un número muy reducido de ejemplos que contienen sólo unas cuantas proposiciones; cuando los autores que no son juristas discuten ejemplos jurídicos tienden a presentar una imagen simplificada de lo que supone un argumento sobre un caso en su conjunto, incluso cuando se trata de casos sencillos ${ }^{63}$.

\section{Y llegamos nuevamente a WIGMORE.}

\subsubsection{El Chart Method y otras alternativas}

ROBERTS afirma que «no se necesita ser un defensor del chart method de WIGMORE para tomar los hechos en serio». Sin duda esto es cierto, pero si «tomar los hechos en serio» ${ }^{64}$ en la enseñanza incluye inculcar habilidades para el análisis de los hechos, como argumenta ROBERTS, tiene que estar claro cuáles son las opciones. Como enfoques alternativos al análisis de hechos, ROBERTS menciona las narraciones y el relato de historias, así como la teoría bayesiana. A su juicio, se trata de enfoques complementarios más que de enfoques opuestos ${ }^{65}$. Sin embargo, la bibliografía que cita consiste en discusiones acerca de estos métodos, más que sobre herramientas para desarrollar habilidades concretas relacionadas con la construcción de argumentos sobre cuestiones de hecho. Otra alternativa puede ser el trabajo de la Escuela de la Argumentación de Amsterdam ${ }^{66} \mathrm{o}$ algunos desarrollos de aplicaciones informáticas como las de $\mathrm{H}$. PRAKKEN, por ejemplo ${ }^{67}$. A mi modo de ver, no obstante, estos enfoques tienden a simplificar demasiado la naturaleza de la argumentación en contextos jurídicos. Y en todo caso, son concepciones bastante parecidas al enfoque de WIGMORE ${ }^{68}$.

62 Existen buenos libros sobre lógica informal, por ejemplo, WALTON, 1989 y 2002, ToULMIN, 1964, pero son pocos los que pretenden enseñar habilidades y técnicas relevantes. Posiblemente el mejor libro siga siendo el de BLACK, 1952. Sobre la «lógica borrosa», vid., ZADEH et al., 1974, y YAGER et al., 1987, así como las críticas de HAACK, 1996.

63 El juicio de Sally Clark es un sorprendente ejemplo de la manera en que incluso los médicos expertos más destacados pueden simplificar excesivamente lo que está en juego en la prueba de una acusación de homicidio (R vs. Clark (Sally) [2003] EWCA Crim 1020), discutido en el simposio titulado «Statistics and Law» en 2005, Significance, 2 (1); DAWID, 2005; BATT, 2004, y DwYER, 2003.

64 RoberTs, 2002, p. 305. Sobre el chart method, vid. TWINING, 2006, p. 12, n. 15.

65 En este punto concuerda con SCHum, 1994, y ANDERSON, SCHum y Twining, 2005.

66 Sobre la Escuela de Amsterdam, vid. FETERIS, 1999a. Para un contraste entre las aplicaciones del enfoque de Amsterdam y el chart method al extraño caso del asesinato producido con un bolígrafo en los Países Bajos (el «Ballpoint case»), vid. Feteris, 1999b, y Dingley, 1999, en MALSCH y NijBOER, 1999, caps. 8 y 9.

67 PRAKKen, 1996 y 2004.

68 Una discusión de los usos y limitaciones de una versión modificada del análisis wigmoreano puede encontrarse en ANDERSOn y TwINING, 1991, Prólogo y pp. 117-131; Twining, 1985, pp. 179-186. Posiblemente el mejor argumento a favor de enseñar el chart method es que funciona. Los autores de Analysis of Evidence, ANDERSOn, SCHUm y Twining, calculan que entre los tres han acumulado más de cincuenta años de experiencia enseñando este enfoque en varias instituciones, principalmente a estudiantes de Derecho, pero también, en el caso de Schum, a analistas de inteligencia e ingenieros. Esta experiencia demuestra que las objeciones tradicionales no son aplicables. Prácticamente todos nuestros estudiantes han considerado que el proceso para aprender el método exige muchos esfuerzos y que es laborioso (el lema de nuestros cursos ha sido «difícil, pero 
Como advierte ROBERTS, el chart method es solamente una entre muchas otras maneras de organizar datos con el propósito de construir un argumento. Otros métodos usuales son las tablas cronológicas, las historias y el método esquemático (la organización de pruebas en función de la fuente de la que provienen, tal y como se lleva a cabo en algunos los libros de litigio en Estados Unidos) ${ }^{69}$. No obstante, ninguno de estos métodos permite organizar el material en forma de un argumento estructurado; por lo general son una ayuda preliminar para construir un argumento, mientras que el chart method representa el argumento en sí. Y si seguimos a THAYER, la argumentación es el contexto en el que se entienden y utilizan mejor las reglas de la prueba.

La queja más común en relación con el chart method es que lleva mucho tiempo y que es muy laborioso. Este argumento tiene algo de solidez, pero necesita ser examinado a la luz de dos distinciones. En primer lugar, la distinción entre dominar una habilidad y aplicarla en la práctica. Aprender a utilizar rigurosamente el chart method es difícil y requiere mucho tiempo. Pero esto también ocurre con cualquier otra habilidad intelectual importante que sea desarrollada en una enseñanza del Derecho de carácter formal. Es posible presentar las cuestiones básicas bastante rápido, pero una habilidad real sólo puede desarrollarse si se refuerza con ejercicios continuos y a través de la experiencia. El dominio de una habilidad supone la comprensión de su utilización y de sus limitaciones. Una vez que se domina una habilidad, es posible decidir cuándo utilizarla de manera informal y cuándo aplicarla rigurosamente. Una vez que se han dominado las habilidades básicas, el método inculca hábitos mentales disciplinados que pueden practicarse rutinariamente de manera informal.

El chart method tiene dos aspectos principales; el análisis macroscópico del caso en su conjunto y el análisis microscópico de fases concretas del argumento del caso. En primer término, el método es una técnica sumamente útil para el manejo de los hechos, para ordenar pruebas y para estructurar argumentos en casos complejos. La estructura del argumento se obtiene trabajando hacia atrás a partir de la última conclusión (la parte superior del diagrama) y organizando las distintas fases del argumento en sectores diferenciados y manejables. La clave consiste en fijar los argumentos del caso en su conjunto en un punto de vista definido, en preguntas claras y en hipótesis o conclusiones formuladas de manera precisa. El trazado de la estructura

divertido»); no obstante, la gran mayoría ha conseguido dominar las técnicas básicas y son muchos los que han elaborado trabajos excelentes. Curiosamente, esta materia ha tenido mejores resultados entre los estudiantes de primer año en la Universidad de Miami, donde es una optativa muy demandada en el segundo semestre. Entre aquellos que se han dedicado a la práctica, muchos han señalado que el enfoque les ha sido de mucha utilidad y algunos consideran que ha sido el curso más útil que tuvieron durante la carrera. Por supuesto, los estudiantes no se pasan el tiempo dibujando elaborados diagramas en casos sencillos, pero las técnicas básicas para ordenar pruebas y construir argumentos pueden convertirse en hábitos mentales importantes y eficaces para manejar casos simples y complejos. Esto tampoco debería sorprender porque el método de WIGMORE es una sistematización de la práctica de los buenos abogados. Algunos ejemplos publicados en los que se ha aplicado el chart method a casos reales son: ABIMBOLA, 2002 (S. LAWRENCE); ANDERSON y GELLER, 2003 (en donde se aplica el análisis wigmoreano a la discusión sobre la subsistencia de la escritura cuneiforme); DingLEY, 1999 (el «ballpoint case» en los Países Bajos), cfr. Feteris, 1999; KAdANe y Schum, 1996 (SACCO y VAnZetTi); Robertson, 1990 (A. A. Thomas); Wigmore (1913) (Hatchett y Umilian). Para ejemplos adicionales, vid., ANDERSON, SCHUM y TWINING, 2006.

69 En la nueva edición de Analysis of Evidence se abordan todos estos cuatro enfoques y se consideran como complementarios. Una aplicación interesante del enfoque simplificado de WigmorE en la preparación de un juicio puede verse en PALMER, 2003. 
básica de un argumento es un mecanismo de simplificación. Incluso en casos muy complejos, el chart method no suele llevar demasiado tiempo, justo al revés: permite ahorrar tiempo ${ }^{70}$.

El segundo aspecto consiste en un análisis preciso y detallado de las fases importantes del argumento. A menudo se percibe el análisis microscópico como un trabajo extremadamente arduo y que lleva mucho tiempo. No obstante, al identificar las fases cruciales o importantes de un argumento y al enfocarse principalmente en ellas, el hábil analista aprende algunos principios básicos de economía. En el ámbito del litigio, «ir a la yugular» significa concentrar nuestro ataque en los puntos más débiles del argumento del oponente o, en sentido positivo, en fortalecer el respaldo de una proposición, lo cual, de conseguirlo, asegurará el éxito. El chart method de análisis es especialmente útil para identificar las proposiciones más importantes de un argumento o de una investigación, para guiar la investigación mediante la identificación de proposiciones potencialmente importantes que son necesarias. La objeción más común de los juristas prácticos es que, en su versión más pura, el método wigmoreano es demasiado laborioso y conlleva mucho tiempo para resultar una herramienta práctica para preparar un juicio ${ }^{71}$. Pero esto sólo ocurre cuando se intenta realizar un diagrama completo del caso en su conjunto. Por lo general el análisis microscópico se puede limitar únicamente a algunas etapas importantes de un argumento.

Estructurar un argumento no necesariamente lleva mucho tiempo y el análisis microscópico preciso puede ser selectivo. Sin embargo, el dominio de las habilidades lleva tiempo. Con todo, subsiste el problema de integrar el análisis de hechos y el Derecho probatorio en un todo coherente. Es evidente que hay cuestiones de prioridades, pero el problema tiene solución. En mi opinión, la solución que ha funcionado mejor es la que se ha adoptado en la facultad de Derecho de la Universidad de Miami, donde la materia de Analyisis of Evidence es una optativa en el primer año de la carrera. Una segunda posibilidad consiste en integrar el análisis de hechos con el análisis jurídico en un curso de metodología jurídica orientado al desarrollo de habilidades. Esta integración permite sentar las bases para poder reforzarlas posteriormente. Si ninguna de estas opciones es viable, los principios de la prueba y las reglas de prueba han de abordarse en un sólo curso, pero en este caso, primero se abordaría el análisis de la prueba y de los hechos en lugar de la relevancia, pero dedicando un poco más de tiempo a este último tema. Esto es lo que ha hecho T. ANDERSON en las primeras tres o cuatro semanas de un curso estándar de Evidence de cuatro créditos en Miami, y de un modo bastante distinto, es lo que llevo a cabo en las primeras cinco o seis semanas

70 Puede resultar problemático construir la parte superior del diagrama cuando hay dudas sobre las normas aplicables o cuando hay múltiples cargos o pretensiones. No obstante, tales problemas se presentan con independencia del método que se adopte. El caso de Louise Woodward (la niñera de Boston), el cual incluye varias gradaciones posibles de homicidio, es un buen ejemplo para explorar estas dificultades [Commonwealth v. Woodward, 7 Mass. L. Reptr. 449 (Mass Super., 1997)], (http://www.courttv.com/trials/woodward/).

71 Por ejemplo, P. MuRPHY afirma lo siguiente: «A pesar de los esfuerzos por presentar el método de Wigmore como una herramienta práctica utilizable... en su versión original, el método wigmoreano supone una pérdida de tiempo innecesaria e impracticable desde el punto de vista del jurista práctico». MURPHY, 2000, 1n. Compárese con el tratamiento más favorable en MuRPHY (ed.), 2003. En la medida en que enseñamos un uso selectivo del chart method y no «el método wigmoreano en su versión original», no existen desacuerdos. 
de curso anual sobre Evidence and Proof en el programa de la Maestría en Derecho en Londres ${ }^{72}$.

A pesar de que algunos temas del Derecho probatorio pueden quedar un tanto ajustados, el enfoque es económico porque (1) los conceptos básicos de la lógica de la prueba y las reglas de pruebas son ampliamente compartidos; (2) pensar en la prueba en términos de razonamiento inferencial y de argumentación establece las bases de una visión thayeriana del Derecho probatorio como un conjunto coherente; (3) una vez que se han expuesto las habilidades básicas, éstas pueden irse reforzando a lo largo del curso, si se considera la relevancia y el análisis de hechos como una parte de otros temas más concretos del Derecho probatorio, y (4) después de algunas dificultades iniciales, la mayoría de los estudiantes considera que se trata de un enfoque interesante y realista.

Este enfoque es eficaz porque al aprender las cuestiones básicas del chart method, los estudiantes comienzan a comprender muchas otras cuestiones. En aproximadamente seis semanas, por ejemplo, he expuesto: a) los conceptos básicos del Derecho probatorio y de la lógica de la prueba; $b$ ) el trasfondo teórico del chart method (incluyendo una comparación con las tablas cronológicas, las narraciones y el método esquemático; c) una aplicación detallada de cada uno de los elementos del protocolo de siete pasos a una cause célebre compleja, y d) la concepción thayeriana y un panorama

72 Sólo a manera de ilustración (dado que hay muchas maneras de llevarlo a cabo), permítanme describir lo que hago en las primeras seis o siete semanas de la parte que doy en el curso de maestría en Londres. El primer paso consiste en dar a los estudiantes un listado de alrededor de veinte conceptos y trabajar con estos conceptos utilizando ejemplos sencillos (tomados de ANDERSON, SCHUm y TwINING, 2005, pp. 10 [«Two murders»] y 40-45 y [una investigación policial imaginaria], explicándolos con dibujos muy sencillos que solamente incluyen cuadrados y círculos como símbolos. Se requieren alrededor de tres horas de clase para dilucidar, explicar y discutir los conceptos más importantes. Como es evidente, el concepto de relevancia es central. Para dilucidar este concepto, en primer lugar se distingue de otros conceptos como el de materialidad, admisibilidad, peso y credibilidad. Posteriormente se dilucida este concepto vinculándolo con las generalizaciones a partir de la experiencia, cadenas de inferencias, convergencia, corroboración y con el —un tanto más complejoproblema de la conjunción que plantea Jonathan Cohen en relación con el caso en su conjunto. Entretanto, los estudiantes han leído una interesante historia, «The Nine Mile Walk» (ANDERSON, ScHum y Twining, 2005, pp. 9-15), la cual ilustra varios puntos, incluyendo la importancia de la abducción y del razonamiento imaginativo. Posteriormente pasamos directamente al chart method, presentado de manera esquemática, sistematizado en el protocolo de siete pasos y explicándolo a partir la misma investigación policial sobre un asesinato. La mejor forma de aprender el método es llevándolo a cabo, por lo que durante las primeras seis semanas se les pide a los estudiantes tres ejercicios escritos que van aumentando de dificultad, alguno de los cuales lo realizan en parejas que representan a un despacho de abogados. Por lo general dedicamos hasta tres semanas de clase para trabajar con el procedimiento de siete pasos en relación con el caso de Reg. v. Bywaters and Thompson (SCHUm utiliza el caso de Sacco y Vanzetti), ANDERSON el caso hipotético de Abel y Archer y uno o dos casos de la Suprema Corte de Estados Unidos. Posteriormente, las lecciones introductorias se refuerzan mediante una discusión mutua con los alumnos de los ejercicios, mini-juicios y una o dos sesiones sobre la relación entre los principios de prueba y el Derecho probatorio. La parte que exige mayor dedicación a los estudiantes es un ejercicio más importante —el análisis completo de un caso complejo (pero que incluye un análisis microscópico selectivo) — que tienen que hacer fuera de clase. Este ejercicio equivale al 35 por 100 de la nota final. No tienen que entregarlo sino hasta el final del año, después de un taller que llevamos a cabo en junio («el día de Wigmore»). En la sección que imparto durante la octava semana, por lo regular tengo tiempo para ocuparme de los relatos, las generalizaciones y los estándares de decisión. Las tres o cuatro semanas posteriores están dedicadas al tema de probabilidad y prueba (impartido por el profesor Ph. DAWID). No se puede esperar que en un curso de licenciatura los estudiantes alcancen un nivel alto en el manejo del chart method y es posible que haya que sacrificar alguna parte (quizá la parte de estadística). En un pequeño curso intensivo de una semana en la Universidad de Puerto Rico en el año 2004, los estudiantes captaron las cuestiones básicas pero hacía falta más tiempo para hacer ejercicios y reforzar otros aspectos. 
general de las reglas de la prueba ${ }^{73}$ y la relación entre los principios de la prueba y las reglas de admisibilidad ${ }^{74}$.

En síntesis, una condición previa para entender el tema de la prueba en el Derecho es entender la lógica de la prueba; una combinación de teoría y práctica permite desarrollar mejor el entendimiento de la prueba; el dominio de los métodos de análisis que involucra el chart method es laborioso y lleva tiempo; aprender las cuestiones básicas lleva menos tiempo. A pesar de su apariencia, si se utiliza de manera inteligente y selectiva, el chart method es una manera eficaz de ocuparse de argumentos complejos sobre cuestiones de hecho y proporciona las bases y un contexto para entender y utilizar las reglas de la prueba.

\subsection{El surgimiento de la prueba como un ámbito interdisciplinar: algunas implicaciones para el Derecho}

Posiblemente el desarrollo más importante en el tema de la prueba en los últimos diez años ha sido el surgimiento de la prueba como un ámbito multidisciplinario con una gran repercusión pública. Son muchos los factores que han contribuido a ello: el análisis de $\mathrm{ADN}$ y los avances en la ciencia forense han estado acompañados por el elevado nivel de científicos forenses en la literatura de ficción y en la televisión. Eventos trágicos como los ocurridos en Europa del Este, Rwanda, Sudáfrica y Latinoamérica han estimulado un enorme interés en la «memoria», no sólo debido a las Comisiones de la Verdad y de Reconciliación y los tribunales penales internacionales, sino también en un buen número de disciplinas académicas ${ }^{75}$. El «giro narrativo» en distintas disciplinas; el aumento de enfoques basados en pruebas en la medicina, en las políticas públicas y en la educación; la búsqueda de «armas de destrucción masiva», los informes sobre Iraq y las investigaciones de HUTTLER y BUTLER, estaban relacionadas centralmente con cuestiones probatorias $^{76}$. Pero han sido especialmente los eventos del 11 de septiembre de 2001 los que han planteado retos fundamentales a los métodos de los servicios de inteligencia para ordenar, interpretar y analizar información. Hay muchos otros ejemplos ${ }^{77}$.

Durante ese mismo periodo ha habido desarrollos en disciplinas académicas que han sido análogos o que han respondido a estos estímulos. No es sorprendente que haya un movimiento que trata de unir todos estos distintos elementos en un solo campo coherente y multidisciplinario. Entre estos esfuerzos destaca el trabajo de D. ScHum, cuyo Evidential Foundations of Probabilistic Reasoning sigue siendo el mejor punto de partida $^{78}$.

73 Vid. Twining, 2006, cap. 5.

74 En la nueva edición de Analysis of Evidence (2005) se pone un énfasis mucho mayor en este último punto y un capítulo completo está dedicado a los principios de la prueba y el Derecho probatorio (cap. 11). Casos como los de DPP v Boardman [1972] AC 421, HL, R v Sang [1980] AC 402, HL, y R v George Joseph Smith [1914-1915] All ER 262, CCA («the Brides in the Bath case») son ejemplos de casos destacados en el Derecho probatorio que también son un vehículo adecuado para encontrar ejercicios sobre la lógica de la prueba. Vid., asimismo, ACORN, 1991, y ANDERSON, 1999.

75 Por ejemplo, Nino, 1996; KROG, 1999; Amadiume y AN-NA'Im, 2000.

76 Hutton, 2004, y ButLer, 2004, analizados en RunCiman, 2004.

77 National Commission on Terrorist Attacks, 2004. Vid. TwINING, 2006, cap. 15.

78 SCHUM, 1994-2001, «Evidence and Inference in History and Law» fue un intento modesto para llevar adelante la agenda de SCHUm. Ahora en Twining y HAmPSHER-Monk (eds.), 2003, cap. 1. 
Es evidente que el Derecho también puede contribuir a estos desarrollos, al igual que puede aprender mucho de ellos ${ }^{79}$. ¿Pero cuál sería su importancia para los cursos sobre la prueba en las facultades de Derecho? Durante el siglo XX, el estudio de la prueba estuvo abierto a una variedad de influencias provenientes de otras disciplinas —algunos ejemplos conocidos son la ciencia forense, la psicología forense, la estadística, la lógica, la teoría narrativa, la inteligencia artificial, la teoría de la complejidad y las aplicaciones informáticas- ${ }^{80}$. Se trata de una tradición bien establecida que se ha trasladado también al ámbito de la enseñanza. El desarrollo de un campo claramente multidisciplinar lleva a plantear, como mínimo, algunas cuestiones de fondo acerca de hasta qué punto las ideas sobre la prueba son transferibles a través de disciplinas, culturas y contextos prácticos distintos. Del mismo modo, amplía enormemente el número de casos, ejemplos concretos y cuestiones que pueden ser dignas de atención para los juristas.

Puede que pase algún tiempo para que sea visible cuáles son las implicaciones de estos desarrollos en la enseñanza de la prueba en el Derecho. Al establecer vínculos con otras disciplinas, el número de ideas y ejemplos concretos a los que se puede acceder es mucho más extenso. Naturalmente, en la enseñanza de la prueba se han utilizado ejemplos no jurídicos y cuasi-jurídicos, como el juicio de Salomón, la piedra de Rosetta, conocidos misterios y problemas históricos, y las historias de Sherlock Holmes, han figurado en los cursos y en los libros sobre la prueba. El repertorio de ejemplos que puede utilizarse en la enseñanza ha aumentado con rapidez. Por ejemplo, tras los sucesos del 11 de septiembre, D. SCHUM ha diseñado una serie de escenarios para capacitar a los analistas de inteligencia - algunos alarmantemente realistas-que pueden utilizarse para enseñar a los estudiantes de Derecho el razonamiento inferencial y el manejo de los hechos. Además de que son interesantes en sí mismos, estos ejemplos no estrictamente jurídicos sirven para reafirmar el mensaje de que el estudio de la prueba en el Derecho está relacionado fundamental e inevitablemente con el estudio de la prueba en general.

\section{(Trad. de Raymundo Gama Leyva)}

\section{BIBLIOGRAFÍA CITADA}

Aвimbola, K., 2002: «Questions and Answers: The Logic of Preliminary Fact Investigation», Journal of Law and Society, 29 (4).

ACORN, A. E., 1991: «Similar Fact Evidence and the Principle of Inductive Reasoning: Making Sense», Oxf. Jo. Leg. Stud., 11 (1).

Allen, R. J., y PARDO, M. S., 2003: «Facts in Law and Facts of Law», International Journal of Evidence and Proof, 7 (3).

Amadiume, I., y Abdullahi, A.-N. (eds.), 2000: The Politics of Memory: Truth, Healing, and Social Justice, London.

Anderson, T., 1999: «The Netherlands Criminal Justice System: An Audit Model of Decisionmaking», en M. MAlsch y J. H. NijbOeR (eds.), Complex Cases: Perspectives on the Netherlands Criminal Justice System, Amsterdam.

79 Sobre las limitaciones del Derecho en este contexto, vid. TwINING, 2006, cap. 15.

80 Cfr. la formulación clásica de Michael, 1948, citada en TwINING, 2006, pp. 16-17. 
Anderson, T., y Geller, M., 2003: «The Last Wedge, Wigmore Meets the Last Wedge, Wigmorean Analysis and the Survival of Cuneiform» en W. L. TWINING e I. HAMPSHER-MONK (eds.): Evidence and Inference in History and Law, Evanston, caps. 3-5.

Anderson, T., y Twining, W., 1991: Analysis of Evidence, Boston-Toronto.

Anderson, T.; Schum, D., y Twining, W. L., 2005: Analysis of Evidence (2. ${ }^{\text {e ed. }), ~ N e w ~ Y o r k . ~}$

Ashworth, A., 1998: The Criminal Process: An Evaluative Study (2. ${ }^{a}$ ed.), Oxford.

BATT, J., 2004: Stolen Innocence: The Story of Sally Clark, London.

BeCHER-Monas, E., 2003: «The Epistemology of Prediction: Future Dangerousness Testimony and Intellectual Due Process», Washington and Lee Law Review, 60 (2).

Binder, D., y Bergman, P., 1984: Fact Investigation, St Paul, Minn.

BlacK, M., 1952: Critical Thinking, Englewood Cliffs, N. J.

Brewer, S., 1998: «Scientific Expert Testimony and Intellectual Due Process'» Yale L. Jo., 107.

BUTLER, Lord R., 2004: «Review of Intelligence on Weapons of Mass Destruction'», HC 898, London (The Butler Report).

Cogan, J. K., 2000: «The Problem of Obtaining Evidence for International Criminal Courts», Human Rights Quarterly, 22 (2).

ChIlds, M., y ElLison, L. (eds.), 2000: Feminist Perspectives on Evidence, London.

ChOO, A., y NASH, S., 2003: «Evidence Law in England and Wales: The Impact of the Human Rights Act 1998», International Journal of Evidence and Proof, 7 (1).

DAMASKA, M., 1986: The Faces of Justice and State Authority, New Haven.

- 1997: Evidence Law Adrift, New Haven.

DAWID, P., 2002: «Bayes's theorem and weighing evidence by juries'», Proceedings of the British Academy, 71.

— 2005: «Statistics and the Law», en K. Tyberg, J. Swenson-Wright y A. Bell (eds.), Evidence, Cambridge.

Dennis, I., 2002: The Law of Evidence (2. ' ed.), London.

DeZALAY, Y., y GARTH, B., 1996: Dealing in Virtue: International Commercial Arbitration and the Construction of a Transnational Legal Order, Chicago.

Dingley, A., 1999: «The Ballpoint Case: A Wigmorean Analysis», en M. Malsch y J. H. NijBOER (eds.), Complex Cases: Perspectives on the Netherlands Criminal Justice System, Amsterdam, cap. 9.

DworkIN, R., 1977: Taking Rights Seriously, London.

- 1986: Law's Empire, London.

DWYER, D., 2003: «The duties of expert witnesses of fact and opinion: R v. Clark (Sally)», International Journal of Evidence and Proof, 7 (4).

Ellison, L., 1998: «Cross-examination in Rape Trials», Crim. L. Rev., 605.

FETERIS, E., 1999a: Fundamentals of Legal Argumentation, Dordrecht.

- 1999b: «What Went Wrong in the Ballpoint Case?», en M. Malsch y J. H. NijbOeR (eds.), Complex Cases: Perspectives on the Netherlands Criminal Justice System, Amsterdam.

Finkelstein, M. O., y LEVIN, B., 2001: Statistics for Lawyers, New York.

GastwirTh, J. L. (ed.), 2000: Statistical Science in the Courtroom, New York.

GolD, N. (ed.), 1982: Essays on Legal Education, Toronto.

HaAcK, S., 1996: Deviant Logic: Fuzzy Logic, Chicago.

- 2003a: Defending Science Within Reason, New York.

- 2003b: «Inquiry and Advocacy, Fallibilism and Finality: Culture and Inference in Science and the Law», Law, Probability and Risk, 2. 
- 2004: «Truth and Justice, Inquiry and Advocacy, Science and Law», Ratio Juris, 17 (1).

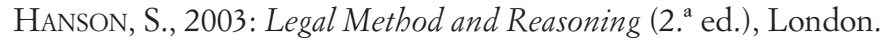

Hawkins, P., y Hawkins, A., 1998: «Lawyers' Probability Misconceptions and the Implications for Legal Education», Legal Studies, 18 (3).

Hirsch, D., 2003: Law Against Genocide: Cosmopolitan Trials, London.

HutTon, Lord B., 2004: Report of the Inquiry into the Circumstances Surrounding the Death of Dr David Kelly CMG, HC 247, London (The Hutton Report).

JACKSON, J., 2005: «The Effects of Human Rights on Criminal Evidentiary Processes: Towards Convergence, Divergence or Realignment?», Modern Law Review, 68 (5).

KadAne, J. B., y Schum, D. A., 1996: A Probabilistic Analysis of the Sacco and Vanzetti Evidence, New York.

Kibble, N., 2004: «Judicial Perspectives on Section 41 of the Youth Justice and Criminal Evidence Act 1999», Research Report for Criminal Bar Association, Aberystwyth.

KIRGIS, P. F., 2004: «Questions of fact in the practice of law: A response to Allen and Pardo's "Facts in law and facts of law"», International Journal of Evidence and Proof, 8 (1).

KroG, A., 1999: Country of my Skull, London.

LEARY, R., 2004: «Evidential Reasoning and Analytical Techniques in Criminal Pre-Trial Fact Investigation», Tesis de Doctorado, University College London.

LEDwOn, L., 2003: «The Poetics of Evidence: Some Applications from Law and Literature», Quinnipiac Law Review, 21.

LEMPERT, R., 2001: «The Economic Analysis of Evidence Law: Common Sense on Stilts», Virginia Law Review, 87 (8).

Llewellyn, K. N., 1960: The Common Law Tradition: Deciding Appeals, Boston.

- (1962: Jurisprudence: Realism in Theory and Practice, Chicago.

MacCormicK, D. N., 1984: «Coherence in Legal Justification» en W. KraWIETZ, H. SCHESKey, G. WinkLeR, y A. Schramm (eds.), Theorie der Normen, Berlin.

- 2005: Rhetoric and the Rule of Law: A Theory of Legal Reasoning, Oxford.

Mack, K. (1998: «Teaching Evidence: Inference, Proof and Diversity», Legal Education Review, 9.

Malsch, M., y Nijboer, J. H. (eds.), 1999: Complex Cases: Perspectives on the Netherlands Criminal Justice System, Amsterdam.

May, R.; Tolbert, D.; Hocking, J.; Roberts, K.; JiA, B. B.; Mundis, D., y Oosthuizen, G., 2001: Essays on ICTY Procedure and Evidence, The Hague.

MichaEL, J., 1948: Elements of Legal Controversy, Brooklyn.

Montrose, J. L., 1968: Precedent in English Law and Other Essays (ed. H. Hanbury), Shannon.

Murphy, P., 2000: Murphy on Evidence (7. ${ }^{\text {a }}$ ed.), London.

- 2001: «Teaching Evidence, Proof, and Facts: Providing a Background in Factual Analysis and Case Evaluation», 51 (4).

- 2003: Evidence, Proof and Facts: A Book of Sources (ed.), Oxford.

Nicolson, D., 1994: «Truth, Reason and Justice: Epistemology and Politics in Evidence Discourse», The Modern Law Review, 57 (5).

- 1997: «Facing Facts: The Teaching of Fact Construction in University Law Schools», International Journal of Evidence and Proof, 132.

NinO, C. S., 1996: Radical Evil on Trial, New Haven.

Palmer, A., 2003: Proof and the Preparation for Trials, Pyrmont, NSW. 
Pardo, M. S., 2000: «Judicial Proof, Evidence and Pragmatic Meaning: Toward Evidentiary Holism», Northwestern University Law Review, 95.

Posner, R., 1999: «An Economic Approach to the Law of Evidence», Stanford Law Review, 51 (6).

PRAKKEN, H., 1996: «A dialectical model of assessing conflicting arguments in legal Reasoning», Artificial Intelligence, 4 (3-4).

- 2004: «Analysing reasoning about evidence with formal models of argumentation», Law, Probability and Risk, 3 (1).

Risinger, D. M., 2004: «Unsafe Verdicts: The Need for Reformed Standards for Trial and Review of Factual Innocence Claims», Houston Law Review, 41.

Roberts, P., 2002: «Rethinking the Law of Evidence», Current Legal Problems, 55.

Roberts, P., y ZucKerman, A., 2004: Criminal Evidence, Oxford.

Robertson, B., 1990: «John Henry Wigmore and Arthur Allan Thomas: an example of Wigmorian Analysis», Victoria University of Wellington Law Review, 20.

RoebucK, D., 2001: Ancient Greek Arbitration, Oxford.

Runciman, W. G. (ed.), 2004: Hutton and Butler: Lifting the Lid on the Workings of Power, Oxford.

Schum, D., 1994/2001: Evidential Foundations of Probabilistic Reasoning, Evanston, I11.

- 1999: «Marshalling Thoughts and Evidence During Fact Investigation», South Texas Law Review 40 (2).

- 2003: «Evidence and Inferences about Past Events: An Overview of Six Case Studies», en W. L. Twining e I. Hampsher-Monk (eds.), Evidence and Inference in History and Law, Evanston, Ill, cap. 1.

Sedley, S., 2005: «The Rock or the Open Sea? Where is the Human Rights Act Heading?», Journal of Law and Society, 32 (1).

ShaPland, J., 1981: Between Conviction and Sentence: The Process of Mitigation, London.

SIEGEL, M., 1994: «A Pragmatic Critique of Modern Evidence Scholarship», Northwestern University Law Review, 88.

Thayer, J. B., 1898: A Preliminary Treatise on Evidence at Common Law, Boston (reimp. 1969).

Toulmin, S., 1964: The Uses of Argument, Cambridge.

TwInING, W., 1997: Law in Context: Enlarging a Discipline (Oxford) (LIC).

- 1985: Theories of Evidence: Bentham and Wigmore, London y Stanford: Weidenfeld and Nicolson.

- 1978: «The Great Juristic Bazaar», Journal of the Society of Public Teachers of Law, 14 (New Series: 185.

- 2002: The Great Juristic Bazaar: Jurists «Texts and Lawyers» Stories, Aldershot.

- 2005: «Have Concepts: Will Travel», International Journal of Law in Context, 1 (1).

- 2006: Retbinking Evidence. Exploratory Essays (2. $\left.{ }^{\mathrm{a}} \mathrm{ed}\right)$, Cambridge.

VAn Kampen, P., 1998: Expert Evidence Compared, Amberes.

WagenaAR, W. A., y Crombag, H., 2005: The Popular Policeman and Other Cases: Psychological Perspectives on Legal Evidence, Amsterdam.

Wagenaar, W. A.; Van Koppen, P. J., y Crombag, H. F. M., 1993: Anchored Narratives: The Psychology of Criminal Evidence, London.

WaLD, P., 2003: «Rules of Evidence in the Yugoslav War Tribunal», Quinnipiac Law Review, 21: 761.

Walton, D., 1989: Informal Logic, New York.

- (2002: Legal Argumentation and Evidence, University Park, Pa. 
Wigmore, J. H., 1913: «The Problem of Proof», Illinois Law Review, 8 (2).

- 1937: The Science of Judicial Proof, as given by Logic, Psychology and General Experience (3. ${ }^{a}$ ed.), Boston.

ZADEH, L. et al. (eds.), 1974: Fuzzy Sets and their Applications to Cognitive and Decision Processes, Berkeley.

Zuckerman, A., 1989: The Principles of Criminal Evidence, Oxford. 